\title{
Caring for Patients Takes Time: Dr. Peabody Says So!
}

\author{
Eugene V. Beresin • Richard Balon • John H. Coverdale • \\ Alan K. Louie • Jane Paik Kim • Laura Weiss Roberts
}

Received: 17 October 2014 / Accepted: 28 October 2014 / Published online: 22 November 2014

(C) Academic Psychiatry 2014

The good physician knows his patient through and through, and his knowledge is bought dearly. Time, sympathy, and understanding must be lavishly dispensed, but the reward is to be found in that personal bond which forms the greatest satisfaction in the practice of medicine.

One of the essential qualities of the clinician is interest in humanity, for the secret of the care of the patient is in caring for the patient.

Francis W. Peabody [1].

The emotional exhaustion of present-day physicians and physicians-in-training is well described and of genuine concern. It is assumed, however, that the origin of exhaustion, cynicism, compassion fatigue, and burnout is patient care or "service" burden. The Accreditation Council for Graduate Medical Education cautions program directors to limit the number of hours in which resident physicians engage in patient care responsibilities. The guidelines imply that caring for patients is intrinsically burdensome - the notion of service is represented as onerous - as mere labor, not as an act of

E. V. Beresin

Harvard Medical School, Boston, MA, USA

R. Balon

Wayne State University, Detroit, MI, USA

J. H. Coverdale

Baylor College of Medicine, Houston, TX, USA

A. K. Louie · J. P. Kim • L. W. Roberts $(\square)$

Stanford University School of Medicine, Stanford, CA, USA

e-mail: RobertsL@stanford.edu professionalism in which our efforts are dedicated to the wellbeing of others and a greater good. The guidelines were introduced, quite correctly, when tragic mistakes in patient care and the exploitative practices of some residency programs were recognized. Nevertheless, Dr. Peabody's remarks in 1927 [1], nearly a century ago, remind us that our aim should not be to limit contact with patients but to provide excellent patient care and to foster great clinical skills in our trainees. Moreover, what if, as Dr. Peabody suggests, by limiting the contact that we have with our patients, we have damaged the deepest source of satisfaction in our work as physicians?

Taken one step further, what if the ever-expanding administrative responsibilities associated with clinical care are diminishing time spent with patients as well as contributing to the emotional exhaustion, depersonalization, and lack of sense of personal achievement of physicians? The time allocated to maintenance of documentation in the electronic medical record, interactions with insurers, and fulfillment of regulatory requirements, among other activities, has grown dramatically over the past decades. A recent RAND study [2] found that physicians experienced many obstacles to providing high-quality care and that these barriers were a major source of dissatisfaction as a professional. Physicians in that study described how the pressure to provide greater quantity of service for a greater number of patients and to engage in extensive administrative activities effectively decreased the time and attention they could spend with each patient, which in turn detracted from the quality of care in some cases. Moreover, limiting hours and contact with patients can set up problems - more mistakes may be precipitated by, for example, multiple hand offs that occur with "shift work" and not really knowing and caring for patients longitudinally, even through a critical episode of illness. A personal sense of fulfillment and integrity is likely compromised when a 
physician is unable to spend time with patients but must instead engage in work that is less meaningful and more isolating or when a physician worries that the system or work environment does not facilitate excellence and trust. Professional integrity can become compromised by the organizational culture within which physicians work when that culture becomes dysfunctional [3]. We are left with the question of what separates professional relationships from contractual relationships under such circumstances.

Physicians, as professionals, have a social contract to heal, educate, and discover. As clinicians and clinical teachers, we see that the foundation for our social contract most often resides in the patient-doctor relationship. Students rapidly learn in the classroom, clinics, and wards that quoting the most elegant evidence from the scientific literature is a gold standard of performance. Although the pursuit of technical knowledge and scientific skills is an essential component of health care, it should not diminish the importance of a full psychological, social, and cultural understanding of the patient living with the condition. Consider the student who understands the chemistry of a metabolic syndrome but lacks deep knowledge of the patient's history; current experience; family background; and social, ethnic, and cultural beliefs that will shape the course of the patient's illness and path toward better health. And although the student's evidence for treatment recommendations may be impeccable, highly supported and valued by the attending physicians, and affirmed by clinical culture, it is clear that an evidence base for a clinical recommendation will not be sufficient by itself to provide excellent care for the patient. Engaging the patient's trust and involvement in treatment and laying the groundwork for the patient's adherence to a clinical regimen take much more than the presentation of rigorous data. Appreciating the social, cultural, and belief systems of the patient helps the doctor understand the meaning of the illness and its treatment for the patient and family, foster a therapeutic alliance, and educate the patient about the illness and the options for effective treatment.

\section{We have to ask ourselves whether medicine is to remain a humanitarian and respected profession or a new but depersonalized science.}

\section{Elisabeth Kübler-Ross [4].}

Indeed, healing involves more than applying current scientific knowledge, employing diagnostic procedures, and implementing therapeutic techniques. Although these elements are instrumental and essential, healing - the art of medicine - provides comfort, reassurance, open and honest communication, respect, and empathy. Promoted by an intimate and sincere patient-doctor relationship, healing also involves affirmation, humor, judicious self-revelation, active listening, altruism, and cultural sensitivity [5]. The professional virtues of integrity, compassion, self-effacement, and self-sacrifice are fundamental to the practice of medicine as a profession [5].

How do physicians become healers and really care for patients? How do we restore and reaffirm the doctor-patient relationship in medicine? How may we do this, within ourselves in our personal self-reflection and self-concept and in the interpersonal field as we develop professional relationships with our patients? With regard to the latter, our current health care system and teaching institutions might utilize means that are pragmatic and concrete, for instance, increasing efficiency in order to free up more time for physicians to spend with patients. Efficiency might be improved by a more user-friendly electronic medical record, utilizing physician extenders or scribes, communicating with patients via Health Insurance Portability and Accountability Act-compliant technologies, expediting "through-put" procedures, decreasing insurance paperwork, and so on. Additionally, the medical system ideally will incentivize "quality time" with patients by introducing more relevant outcome metrics and increasing reimbursement for cognitive services. These are systems-based solutions that will enhance the ability of physicians collectively to make the doctor-patient relationship a greater priority of healthcare into the future. These practices are external to the physician and provide the environmental provision for patient care.

Internal solutions are those shaped and integrated into our ethos and are under the control of the individual physician. Each physician must learn and hone the art of medicine. For instance, empathy is a capacity that may be deepened with practice. In medicine, empathy is essential for healing, yet its expression must be disciplined to avoid excess that results in loss of objectivity, burnout of the physician, and counterproductive overindulgence of the maladaptive traits of a difficult patient. A balance need be maintained; as Dr. Peabody wrote, "The treatment of a disease must be completely impersonal; the care of a patient must be completely personal" [1]. Leif and Fox [6] described this process as a developmental aspect of becoming a doctor in assuming a position of "detached concern"-having enough distance from a patient to care objectively while being emotionally attuned to the patient.

The loss of empathy results in impersonal treatment of patients. This loss may occur in the context of physician burnout after many years of practice, and yet, it is also seen as early as the third year of medical school [7] and certainly during internship and residency. External pressures (e.g., long work hours, excessive workload) are often blamed for the decrease in empathy, but one should not underestimate the possible role of intrapsychic, that is, internal forces. Physicians face emotional circumstances frequently - death of a patient, suffering that does not respond to treatment, dealing with child-abusing parents, the terror of a psychotic experience, and the like. Physicians also worry about making errors or bearing the guilt of having made an error and being exposed to malpractice claims. In such trying moments, retreating from 
these emotions and just focusing on the science of medicine may be of defensive and self-preserving value to the physician. Healing others at these times requires not withdrawing personally, however, and instead forging an emotional connection with the patient to overcome these circumstances.

The personal sustenance provided by the doctor-patient relationship thus is not without personal investment and risk on the part of the physician. Complaining, for example, about perpetual sleep deprivation and burdensome paperwork and not finding time for patients may be emotionally "safer" for an intern in comparison with enduring these feelings. In so doing, unfortunately, the opportunity for learning the art of medicine and its gratification is necessarily compromised.

Internal solutions are relatively abstract, like the regulation of empathy - titrating it for optimal balance, sensitivity, and tolerability. These solutions require a lifetime of clinical experience and deliberate practice of the art of medicine. Two modern movements in medicine and medical education, mindfulness and wellness, support and operationalize this type of practice.

Mindfulness, or reflective practice, can restore and bring new strength to the physician. As Epstein [8] wrote:

Mindful practitioners attend in a nonjudgmental way to their own physical and mental processes during ordinary, everyday tasks. This critical self-reflection enables physicians to listen attentively to patients' distress, recognize their own errors, refine their technical skills, make evidence-based decisions, and clarify their values so that they can act with compassion, technical competence, presence, and insight.

Epstein added, "Mindful practice extends beyond examining the affective domains and involves critical reflection on action, tacit personal knowledge, and values in all realms of clinical practice, teaching, and research" [8]. To be able to reflect on one's clinical practice, to practice mindful medicine, the centrality of the doctor-patient relationship emerges naturally. Reflective practice causes us to take more time with the patient and think about the patient and learn from the patient. In following the advice of Dr. Peabody and in engaging in reflective practice, we affirm our identity as healer, and we elevate the importance of patients and the physician-patient relationship. Mindfulness adds another dimension to the existing medical education literature concerning reflection-on-action $[9,10]$ and its more recent implementation in medical training through self-reflective writing and portfolios. These exercises bring mentoring, peer advising, and external data into the self-reflective process.

A second positive movement in modern medicine that promises to bring greater focus on the physician-patient relationship is the effort to support wellness and diminish sources of impairment among medical students, residents, and physicians. When a physician or physician-in-training seeks health care, the dual role as clinician and patient does much to foster empathy and compassion, as reflected in narrative accounts and empirical studies [11]. As Robert Klitzman [12] wrote in the opening lines of his book, When Doctors Become Patients, the experience is very compelling.

At 8:30 a.m. on September 11, 2001, from her office on the $105^{\text {th }}$ floor of the World Trade Center, my sister Karen called her best friend. No one ever heard from Karen again.

Over the next few weeks, I helped organize a memorial service and a fellowship in her name, signed a death certificate, and packed up all of her belongings. Then my body gave out. For three months, I could not sleep. As a result, I had a flu that would not leave me. I could not get out of bed, and was no longer interested in reading books, seeing movies, or listening to music. Yet I was surprised when friends told me they thought I was depressed.

"No, I am just sick," I said, resisting the idea. I was a psychiatrist, but suddenly, for the first time in my life, had physical symptoms of depression, and was amazed at the experience - how much it was more bodily than emotional. My body had just given way beneath me.

For the first time, I fully appreciated what my patients had to undergo, and how hard it is to put the experience of depression into words. ... I felt weak and ashamed, and began to appreciate, too, the embarrassment and stigma my patients felt.

The experience of being on "both ends of the stethoscope" is transformative and affirms the centrality of the doctorpatient relationship.

In this issue of Academic Psychiatry, several papers examine health, well-being, and self-care of medical students and residents [13-26]. The set includes ten data-based reports. In a single-site study, Al-Mateen et al. [13] found that out of 64 third-year medical students, the majority experienced mental health concerns and psychological distress and developed symptoms from vicarious traumatization while in their psychiatry or surgery clerkships. In a study of 105 first-year medical students by Zanardelli et al. [14], attributes such as gender appeared to shape attitudes toward counseling, and such attitudes were associated with well-being. In a study of 133 psychiatry residents who were in personal psychotherapy, Kovach et al. [15] found that most reported their motivation to be improved self-awareness and self-reflection. The most common reason for not seeking psychotherapy was the lack of time and finances. In another study ( $n=183$ medical students), Gold et al. [16] revealed that the majority of students endorsed stress, which was related to self-reported unmet health needs. Moreover, evidence of unsought health care emerged: more than a third of students did not have a primary care provider, a third of students did not seek health care when sick, and a third of students reported having mental health 
needs for which they did not seek care. Finally, a study led by two of the authors (LWR, JPK) [17] revealed that out of 155 residents at one institution, most had sought health care, very commonly informally through "curbside consultation." Many residents had written prescriptions for themselves, for other trainees, and, astonishingly, for attending physicians. Overall, pressure related to their busy schedules was the major driver behind this pattern of informal care-seeking practices. Time, again, emerged as a central theme in this empirical work that sought to understand the experience of physicians and physicians-in-training related to self-care.

Three papers focused on interventions to address wellness of trainees. Seritan et al. [18] introduced a type of educational intervention that included broad involvement of stakeholders involved in medical education and aimed to improve access to care and mental health service utilization. Wasser [19] designed and pilot-tested an educational intervention among 13 psychiatry residents that aimed to improve trainees' abilities to increase awareness of safety and violence risk in the conduct of the psychiatric interview and found remarkable improvement in safety concerns among residents. A report by Grant et al. [20] examined a range of strategies ascertained through structured interviews of 32 UK medical schools to support medical students with mental health concerns and developed a conceptual model of support to compare interventions across providers. These reports complement a systematic review by Williams et al. [21], wherein a variety of burnout interventions were assessed and described.

Other papers examine the role of mentorship and selfreflection in career advancement and spirituality. Sciutto et al. [22] reported novel aspects of a formal resident advisory program intended to guide residents through developmental milestones - such programs are increasingly common in medical schools yet rarely reported in residency. A commentary by Lipsitt [23] offered a framework to consider efforts to promote self-awareness and a context to minimize risk of illness and impairment. Soklaridis et al. [24] evaluated a formal mentorship program for second-year psychiatry residents at an academic institution to better understand resident attitudes toward the role of mentorship in the facilitation of their growth. Perhaps more encouraging is a study by Krägeloh et al. [25] on spirituality in 275 medical students, which found that hope and optimism, regardless of religious affiliation, were linked to higher reported quality of life.

The relationship between physician and patient is central to the profession of medicine. It is our responsibility, and it is our sustenance as healers. We suggest that the current framing of "service" and "duty" may place physicians and physicians-intraining in opposition to patients, rather than foster a sense of responsibility, fulfillment, and joy in the role of caring for an ill person. This framing does not facilitate trust in our profession, may have unexpected negative effects in clinical care practice, and has contributed to the demoralization of our field. Mindfulness and self-care practices are two positive movements in modern medicine that we believe will help restore the centrality of the physician-patient relationship, with salutary effects, we believe, for patients and for physicians and physicians-in-training. Recognizing the value in sitting with patients, understanding them, and spending time with them - all the while, reclaiming our role as healers and as members of the profession of medicine - is so very important at this time in the history of medicine. We raise this issue of time for reflection, for self-care, and especially for sitting with our patients, with Dr. Peabody's remarks resonating in our thoughts and enveloping our hearts, in the hope that the true sense of duty to our patients will never be lost.

Disclosures On behalf of all the authors, the corresponding author states that there are no conflicts of interest.

\section{References}

1. Peabody F. The care of the patient. JAMA. 1927;88:877-22.

2. Friedberg MW, Chen PG, Van Busum KR, Aunon FM, Pham C, Caloyeras JP, et al. Factors affecting physician professional satisfaction and their implications for patient care, health systems, and health policy. RAND Health, 2013. Available at http://www.rand.org/pubs/ research reports/RR439.html. Accessed July 23, 2014.

3. Chervenak FA, McCullough LB. The diagnosis and management of progressive dysfunction of health care organizations. Obstet Gynecol. 2005;105:882-7.

4. Kübler-Ross E. On death and dying. New York: Macmillan; 1969.

5. Coverdale J, Balon R, Roberts LW. Cultivating the professional virtues in medical training and practice. Acad Psychiatry. 2011;35: $155-9$.

6. Leif HI, Fox RC. Training for 'detached concern' in medical students. In: Leif HI et al., editors. The psychological basis of medical practice. New York: Harper and Row; 1963. p. 12-35.

7. Hojat M, Vergare MJ, Maxwell K, Brainard G, Herrine SK, Isenberg GA, et al. The devil is in the third year: a longitudinal study of erosion of empathy in medical school. Acad Med. 2009;84(9):1182-91.

8. Epstein RM. Mindful practice. JAMA. 1999;282:833-9.

9. Schon DA. The reflective practitioner: how professionals think in action. New York: Basic Books; 1983.

10. Boud D, Keogh R, Walker D. Reflection: turning experience into learning. Oxford: Routledge; 1985.

11. Roberts LW, Warner TD, Moutier C, Geppert CMA, Green Hammond KA. Are doctors who have been ill more compassionate? Attitudes of resident physicians regarding personal health issues and the expression of compassion in clinical care. Psychosomatics. 2011;52:367-74.

12. Klitzman R. When doctors become patients. New York: Oxford University Press; 2007.

13. Al-Mateen C, Linker JA, Damle N, Hupe J, Helfer T, Jessick V. Vicarious traumatization and coping in medical students - a pilot study. Acad Psychiatry. 2014. doi:10.1007/s40596-014-0199-3.

14. Zanardelli G, Sim W, Borges N, Roman B. Well-being in first year medical students. Acad Psychiatry. 2014. doi:10.1007/s40596-0140189-5.

15. Kovach J, Dubin W, Combs C. Use and characterization of personal psychotherapy by psychiatry residents. Acad Psychiatry 2014. doi: 10.1007/s40596-014-0219-3. 
16. Gold JA, Johnson B, Leydon G, Rohrbaugh RM, Wilkins KM. Mental health self-care in medical students: a comprehensive look at help seeking. Acad Psychiatry. 2014. doi:10.1007/s40596-0140202-z.

17. Roberts LW, Kim JP. Informal health care practices of residents: "curbside" consultation and self-diagnosis and treatment. Acad Psychiatry. 2014. doi:10.1007/s40596-014-0170-3.

18. Seritan AL, Rai G, Servis M, Pomeroy C. The office of student wellness: innovating to improve student mental health. Acad Psychiatry. 2014. doi:10.1007/s40596-014-0152-5.

19. Wasser TD. How do we keep our residents safe: an educational intervention. 10.1007/s40596-014-0212-x.

20. Grant A, Rix A, Mattick K, Winter P, Jones D. Support for medical students with mental health problems: a conceptual model. Acad Psychiatry. 2014. doi:10.1007/s40596-014-0154-3.
21. Williams DM, Tricomi G, Gupta J, Janise A. Efficacy of burnout interventions in the medical education pipeline. Acad Psychiatry. 2014. doi:10.1007/s40596-014-0197-5.

22. Sciutto M, Levenson J, Perez A, Oquendo MA, Arbuckle M. Developing an advisor program for psychiatry residents. 10.1007/ s40596-014-0153-4.

23. Lipsitt D. Developmental life of the medical student: curriculum considerations. 10.1007/s40596-014-0182-z.

24. Soklaridis S, Lopez J, Charach N, Broad K, Teshima J, Fefergrad M. Developing a mentorship program for psychiatry resident. 10.1007/ s40596-014-0163-2.

25. Krägeloh CU, Henning MA, Billington R, Hawken SJ. The relationship between quality of life and spirituality, religiousness, and personal beliefs of medical students. Acad Psychiatry. 2014. doi:10. 1007/s40596-014-0158-z. 